\title{
Electrochemical Bubbling Transfer of Graphene Using a Polymer Support with Encapsulated Air Gap as Permeation Stopping Layer
}

\author{
Jie Sun,, ${ }^{1,2}$ Shigui Deng, ${ }^{1}$ Weiling Guo, ${ }^{1}$ Zhaoyao Zhan, ${ }^{2}$ Jun Deng, ${ }^{1}$ Chen $X u,{ }^{1}$ Xing Fan, \\ Kun Xu, ${ }^{1}$ Wang Guo, ${ }^{1}$ Yang Huang, ${ }^{1}$ and Xin Liu ${ }^{1}$ \\ ${ }^{1}$ Key Laboratory of Optoelectronics Technology, Beijing University of Technology, Beijing 100124, China \\ ${ }^{2}$ Mikroteknologi och Nanovetenskap, Chalmers Tekniska Högskola AB, 41296 Göteborg, Sweden
}

Correspondence should be addressed to Jie Sun; jie.sun@chalmers.se and Weiling Guo; guoweiling@bjut.edu.cn

Received 17 November 2015; Revised 28 February 2016; Accepted 6 March 2016

Academic Editor: Egon Pavlica

Copyright ( 2016 Jie Sun et al. This is an open access article distributed under the Creative Commons Attribution License, which permits unrestricted use, distribution, and reproduction in any medium, provided the original work is properly cited.

Electrochemical bubbling transfer of graphene is a technique with high industrial potential due to its scalability, time- and costeffectiveness, and ecofriendliness. However, the graphene is often damaged due to the turbulence and the trapped bubbles formed by the direct $\mathrm{H}_{2} \mathrm{O}$ and $\mathrm{H}^{+}$permeation through the supporting polymer. We invent a graphene mechanical support of polyethylene terephthalate foil/plastic frame/poly(methyl methacrylate) sandwich, with an encapsulated air gap as the permeation stopping layer. The graphene damage is drastically reduced, as confirmed by the morphology and structural and electrical characterization, ultimately improving the controllability/reproducibility of the bubbling transfer of graphene and other two-dimensional materials.

\section{Introduction}

By virtue of its properties, for example, the ultrahigh carrier mobility, electrical and thermal conductivity, and broad wavelength-range optical transmittance, graphene is positioned among the most important electronic materials. Currently, it is at the critical stage of the transmission from academic labs to actual applications. According to the roadmap [1], optoelectronic devices are among the most immediate applications of graphene, for example, transparent electrodes in light emitting diodes [2] and solar cells [3]. In this year, commercial cellphones integrating graphene based touch screen are launched [4]. The best technique to synthesize graphene for these electronic applications is chemical vapor deposition (CVD), where metal foil is used as the catalyst and graphene is synthesized from gas precursors at high temperature. $100 \mathrm{~m}$ long graphene has been realized by Sony by this means [5]. A group of other two-dimensional (2D) materials, for example, h-BN and $\mathrm{MoS}_{2}$, have also been identified. Most of them can be prepared by CVD as well, a scalable and semiconductor industry compatible technology.
Nevertheless, 2D materials usually need to be transferred to target substrates for applications and basic research, as the direct synthesis on insulators by noncatalytic CVD is not yet mature [6]. Take CVD graphene as an example; typically, $\mathrm{Cu}$ catalyst is used and needs to be etched off upon transferring the graphene to insulators [7]. It dramatically increases the cost of raw material and poses a threat to the environment for the risk of heavy metal pollution. In a conservative estimation, the world's annual touch screens production is 3 $\times 10^{7} \mathrm{~m}^{2}$. When they are to be produced by CVD graphene on $50 \mu \mathrm{m}$ thick $\mathrm{Cu}$, more than $10^{4}$ tons' ultrapure $\mathrm{Cu}$ will be used. This value may be underestimated because if multilayer graphene is used, the $\mathrm{Cu}$ consumption is multiple.

Recently, a transfer method by electrochemical bubbling is proposed by Wang et al. [8], Gao et al. [9], and our group [10]. A flexible polymer mechanical support is coated to the graphene/metal foil used as the cathode in a water electrolysis cell. Typically, $\mathrm{NaOH}$ is used as the electrolyte in the aqueous solution. Hydrogen bubbles are generated and squeezed into the graphene-metal interface to mechanically delaminate the graphene/polymer from the metal. Afterwards, the film is 


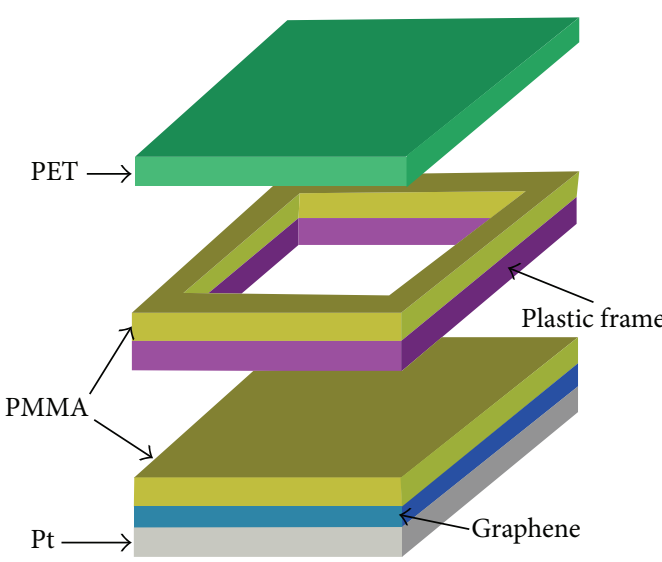

(a)

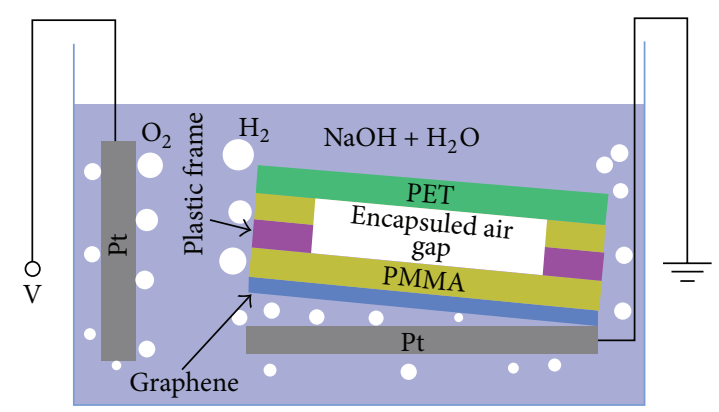

(b)

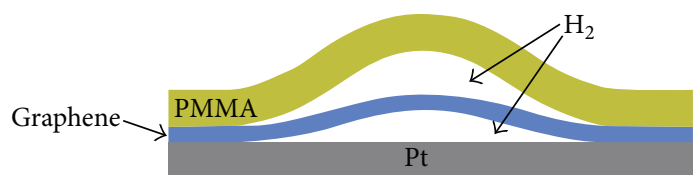

(c)

FIGURE 1: Schematic illustrations of the transfer using a polymeric mechanical support with an encapsulated air gap permeation stopping layer. (a) Sandwiched structure of the support consisting of PET foil, a plastic frame, and a spin-coated PMMA layer on top of the graphene/Pt. The polymeric layers are stuck to each other by using PMMA "glue" on a hotplate. (b) Typical water electrolysis cell composed of Pt anode, $\mathrm{NaOH}$ solution, and a cathode made of the material complex. The encapsulated air gap and the PET foil entirely stop the permeation of $\mathrm{H}_{2} \mathrm{O}$ and $\mathrm{H}^{+}$. (c) When a simple PMMA layer is used as the supporting layer, $\mathrm{H}_{2} \mathrm{O}$ and $\mathrm{H}^{+}$can penetrate it and reach the graphene interfaces. These particles can arrive at the interface much earlier than those coming from the edges along the graphene-Pt interface, leading to trapped $\mathrm{H}_{2}$ bubbles in the center. The strain therein is one of the main causes of the graphene damage.

placed on the target wafer (adhered by van der Waals force) and the polymer is dissolved. The bubbling procedure can delaminate 1-inch graphene from $\mathrm{Cu}$ in a few minutes or quicker [10], whereas in etching transfer usually hours are needed to dissolve the $\mathrm{Cu}$. The catalyst is $\sim 100 \%$ reusable, and it is free of heavy metal emission.

The electrochemical bubbling is a strong candidate for the mainstream transfer technique in graphene industry. Nevertheless, it is not yet widely adapted. One of the main reasons is that the graphene after transfer often shows some tears, wrinkles, and holes. In comparison, the metal-etching-based transfer usually results in less damage. Currently, there is no consensus on the origin of the relatively low controllability and reproducibility of the bubbling transfer. However, we believe the reason is twofold. First, the process is violent due to the many bubbles and the corresponding turbulence in the solution. We have suggested a plastic-frame-based technique where the film is firmly held throughout the process [10] and hence will not be strongly affected by the turbulence. The second factor, which has unfortunately been overlooked so far, is the direct permeation of the $\mathrm{H}_{2} \mathrm{O}$ molecules and $\mathrm{H}^{+}$ ions through the thin polymer and graphene [11]. As seen later, the consequence is that gas bubbles can appear earlier in the center than at the four edges. The trapped bubbles induce large strains and are responsible for the graphene damage. In this letter, we encapsulate a thin layer of air in the polymeric support used in the transfer. The direct permeation of any particles in the solution through the polymers is completely stopped. $\mathrm{H}_{2}$ bubbles can only form at the sample edges and subsequently squeeze into the graphene-metal interface and advance towards the center. Since the polymer support is also thick enough to hold the film against turbulence, the defect in graphene introduced during the bubbling is drastically reduced, as compared with the case without the encapsulated air permeation stopping layer. Morphology observation, Raman spectroscopy, and electrical measurement all indicate that the transferred graphene is of good quality. The technology can be extended to other 2D materials as well, helping accelerate the industrialization of the time- and cost-effective and ecofriendly electrochemical bubbling method.

\section{Materials and Methods}

Monolayer graphene is deposited on Pt foils (99.999\%, $1.2 \times$ $1.2 \mathrm{~cm}^{2}, 100 \mu \mathrm{m}$ thick) in a cold wall CVD system (Aixtron). It is annealed at $1050^{\circ} \mathrm{C}$ for $5 \mathrm{~min}$ in $1000 \mathrm{sccm} \mathrm{H}_{2}$ and $10 \mathrm{sccm}$ Ar. The growth time is merely $5 \mathrm{~min}\left(5 \mathrm{sccm} \mathrm{CH}_{4}\right.$, $1000 \mathrm{sccm} \mathrm{H}_{2}$, and $10 \mathrm{sccm}$ Ar) followed by quench cooling to suppress the carbon segregation and hence multilayer graphene formation [12]. After CVD, the graphene/Pt is spincoated thin poly(methyl methacrylate) (PMMA) (1000 rpm, $160^{\circ} \mathrm{C}$ baking for $10 \mathrm{~min}$ ) as a mechanical support. As shown in Figure 1(a), a plastic frame (thickness $175 \mu \mathrm{m}$ ) is prepared, where on one side we drop-coat PMMA and cure it at $160^{\circ} \mathrm{C}$ for $5 \mathrm{~min}$. Then, polyethylene terephthalate (PET) foil of $77 \mu \mathrm{m}$ thickness is attached to the frame (Figure $1(\mathrm{a})$ ) on 
a hotplate where the PMMA is heated to above the glass transition temperature $\left(T_{g} \approx 105^{\circ} \mathrm{C}\right)$. Here, the PMMA serves as a clean, convenient, and, most importantly, water-proof glue. Subsequently, the PET foil and frame are attached to the PMMA/graphene/Pt in the same manner (Figure 1(a)). The entire mechanical supporting structure is now finished.

The material complex is immersed in $0.25 \mathrm{~mol} / \mathrm{L} \mathrm{NaOH}$ solution and used as the cathode (Figure 1(b)). The anode is also Pt foil, which is chemically inert to avoid complications in the analysis. The current is $1 \mathrm{~A}$. The half equations are written as

$$
\begin{aligned}
& 4 \mathrm{H}_{2} \mathrm{O}+4 \mathrm{e}^{-}=2 \mathrm{H}_{2}+4 \mathrm{OH}^{-} \text {(cathode), } \\
& 2 \mathrm{H}_{2} \mathrm{O}-4 \mathrm{e}^{-}=\mathrm{O}_{2}+4 \mathrm{H}^{+} \text {(anode). }
\end{aligned}
$$

When the graphene and the support structure are completely separated from the underlying Pt, the sample is picked up and rinsed carefully in deionized water. Note that the support makes it easy to handle the graphene sample simply using a tweezer. Afterwards, the PET foil is detached from the frame in water. Since they are sticking to each other only at the edges, a gentle force is enough to peel off the plastic foil. Subsequently, there are two methods to handle the graphene. One can pick up the graphene/PMMA held by the frame and place it on the target substrate ( $\mathrm{Si}$ with $\sim 300 \mathrm{~nm} \mathrm{SiO}{ }_{2}$ ). After drying, the graphene/PMMA is separated from the frame by cutting through the graphene/PMMA along the inner edges of the frame using a cleaving knife. Alternatively, one can already cut off the graphene/PMMA from the frame while it is in water. The graphene/PMMA is floating on the liquid, where surface tension stretches the film and flattens it. The film is then fished up by the target substrate and dried. In both cases, the PMMA/graphene/new substrate is baked at $160^{\circ} \mathrm{C}$ for 10 min to expel the remaining $\mathrm{H}_{2} \mathrm{O}$ and improve the adhesion. The PMMA is removed by acetone at $50^{\circ} \mathrm{C}$.

\section{Results and Discussion}

Why do we bother to encapsulate a layer of air (slightly thicker than the thickness of the frame) in between the PET foil and the graphene/PMMA (Figure 1(a))? PMMA is hydrophilic and transparent to water permeation (diffusion constant up to $5 \times 10^{-10} \mathrm{~cm}^{2} / \mathrm{s}$ ) [13]. Although perfect graphene is tight to all atoms and molecules under ambient conditions [14], since CVD graphene has $\mu \mathrm{m}$-sized crystallites [15], the grain boundaries and some lattice defects provide pathways for the $\mathrm{H}_{2} \mathrm{O}$. Recently, it is discovered that protons, an intermediate case between electrons (which can tunnel through atomic sheets) and atoms, are small enough to pass through the dense electronic clouds of graphene and penetrate with a high rate [16]. Apparently, PMMA is not an effective barrier for $\mathrm{H}^{+}$ions either. At $1000 \mathrm{rpm}$, our PMMA is just $\sim 0.5 \mu \mathrm{m}$ thick. As shown in Figure 1(c), if no encapsulated air permeation stopping layer is employed, it is not surprising that some $\mathrm{H}_{2} \mathrm{O}$ and $\mathrm{H}^{+}$can reach the graphene and $\mathrm{Pt}$ much earlier than those coming sideways. Some of the $\mathrm{H}_{2} \mathrm{O}$ decomposes and produces $\mathrm{H}^{+}$at the interfaces: $\mathrm{H}_{2} \mathrm{O}=\mathrm{H}^{+}+\mathrm{OH}^{-}$. Together with those protons that are directly transported through the PMMA to the interfaces from the solution, these $\mathrm{H}^{+}$ions are reduced to hydrogen atoms, because the graphene and $\mathrm{Pt}$ are both well conducting and can provide electrons. Thus, trapped hydrogen bubbles are produced at the interfaces (Figure $1(\mathrm{c})$ ). The gases are formed more easily at the graphene-Pt interface as compared with the graphene-PMMA interface, due to the fact that many metals, especially $\mathrm{Pt}$ and $\mathrm{Cu}$, are catalysts for hydrogen molecule evolution: $\mathrm{H}+\mathrm{H}=\mathrm{H}_{2}$ [17]. Anyhow, the trapped gases separate the graphene from the Pt and/or the PMMA, leading to quasi-free-standing graphene with large strains. At least partly, it accounts for the graphene damage. Indeed, the earlier formation of $\mathrm{H}_{2}$ bubbles in the center compared to the edges can be frequently observed in our experiments. In this work, the bubbling process lasts $\sim 20 \mathrm{~min}$. When no air gap is used, the delamination only takes a few min [10-12]. The big difference suggests that the bubbles formed in the center by the direct particle permeation through the PMMA are one of the major driving forces for the delamination. However, that efficiency in time is at the expense of the graphene quality, and we prefer to have the bubbles only form at the edges in this letter. We have conducted an investigation of the effect of PMMA thickness on the final graphene morphology after transfer [11]. Not unexpectedly, a direct correlation is found, where a thicker spin-coated PMMA dramatically reduces the pores in graphene. An even thicker PMMA or adhering thick PET foil (e.g., >100 microns) onto the PMMA thoroughly stops the permeation. However, due to the large thickness, the mechanical flexibility is sacrificed, making it difficult for the bubbles to reach the sample center from the edges. Furthermore, even if the graphene is eventually delaminated, it is hard to attach to the new substrate conformally because of the rigidity of the thick support, which translates into pores and ripples in the graphene after support removal. Therefore, we develop the permeation stopping technique in Figure 1. The air gap, together with the PET foil atop, completely stops the $\mathrm{H}_{2} \mathrm{O}$ and $\mathrm{H}^{+}$from penetrating the PMMA. The graphene can only be delaminated starting from the edges. Since the air gap and the thin PMMA are very flexible, the bubbles easily advance to the center and completely detach the sample. It is a facile method to improve the controllability and reproducibility of the bubbling transfer of $2 \mathrm{D}$ materials.

Figure 2(a) is a typical photograph of a graphene film transferred by the above-described method to a silicon substrate with $\sim 300 \mathrm{~nm} \mathrm{SiO}{ }_{2}$ layer. The square shaped graphene is approximately $1 \mathrm{~cm} \times 1 \mathrm{~cm}$ in size and clearly visible to the naked eye. Figures 2(b) and 2(c) show a comparison of the optical micrographs of two graphene items transferred to $\sim 300 \mathrm{~nm} \mathrm{SiO}_{2} / \mathrm{Si}$ by electrochemical bubbling in $\mathrm{NaOH}$, where Figures 2(b) and 2(c) are processed with and without the air permeation stopping layer, respectively. Evidently, the graphene in Figure 2(b) is very flat and smooth. In contrast, in Figure 2(c), the graphene has a number of holes and wrinkles, as indicated by the arrows. Note that, in our graphene characterization, only typical results are shown in this letter. Even without the encapsulated air gap, sometimes the graphene is nearly pore-free. However, it is much more frequent that nice results are achieved upon adding the stopping layer. According to our statistics, the success rate for achieving good transfers is approximately two times higher compared to the 

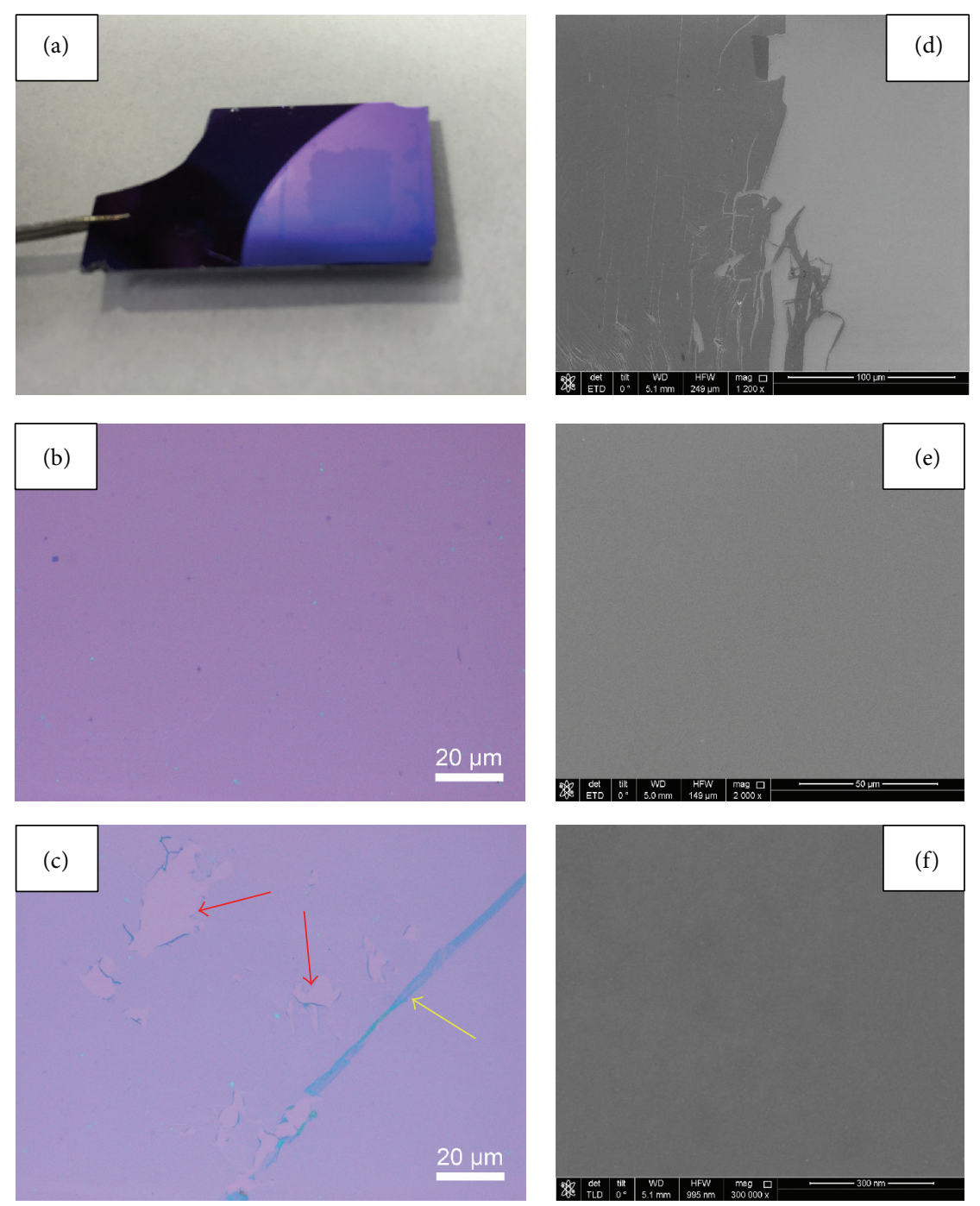

FIGURE 2: Images of typical graphene transferred to $\sim 300 \mathrm{~nm} \mathrm{SiO}_{2}$ on $\mathrm{Si}$. (a) is an ordinary photograph showing $\sim 1 \mathrm{~cm} \times 1 \mathrm{~cm}$ graphene. (b) and (c) are optical microscopy images for graphene transferred by (b) air-gap-assisted and (c) air-gap-free techniques. The arrows indicate the pores and ripples. (d-f) are SEM images, where (d) is taken at the graphene edge. (e) and (f) are taken at low and high magnifications, respectively. The scale bars in $(\mathrm{d}-\mathrm{f})$ are $100 \mu \mathrm{m}, 50 \mu \mathrm{m}$, and $300 \mathrm{~nm}$, respectively.

case without the air gap. Figures 2(d)-2(f) show scanning electron microscopy (SEM) images taken from the samples after the transfer. Figure 2(d) is taken at the graphene edge where a clear contrast between the graphene and the uncovered area is seen. Some mechanical damage at the graphene edge occurs while removing the plastic frames. In the interior of the sample, the graphene appears rather integrated, as evidenced by Figures 2(e) and 2(f), which is captured at low and high magnifications, respectively. In these micrographs, no apparent defects and damage are detected, indicating the feasibility of the method for transferring large area graphene. Figure 3(a) is an atomic force microscopy (AFM) image of the graphene on $\mathrm{SiO}_{2} / \mathrm{Si}$ from air-gap-assisted transfer. We intentionally include an uncovered area to generate some contrast. The boundary of the graphene is indicated by arrows, where the left part is the graphene. Apart from a few particles (possibly introduced by the electrolyte), the graphene is very uniform and as smooth as the uncovered substrate, indicating its high quality. Figure 3(b) shows a Raman spectrum of the graphene measured after the air-gap-assisted transfer to $\mathrm{SiO}_{2} / \mathrm{Si}$. The $2 \mathrm{D}: \mathrm{G}$ ratio is as high as 3.13. Together with a 2D peak full width at half maximum of $32.9 \mathrm{~cm}^{-1}$, it implies that the graphene is a monolayer with high crystalline quality. The small $\mathrm{D}$ peak suggests that the extra defects induced during transfer are negligible. In order to further check the uniformity of the graphene, Raman mapping is performed, as summarized in Figure 4. The three characteristic peaks of the graphene are plotted for the mapped area of $10 \mu \mathrm{m} \times 11 \mu \mathrm{m}$. It can be seen that the graphene is reasonably uniform. The yet present fluctuation in the peak intensities is due to the growth itself and the transfer procedure. The last panel in Figure 4 plots the $2 D / G$ ratio, where an average value of $\sim 2$ is achieved, indicating that the transferred graphene is largely a monolayer with rather good quality. 


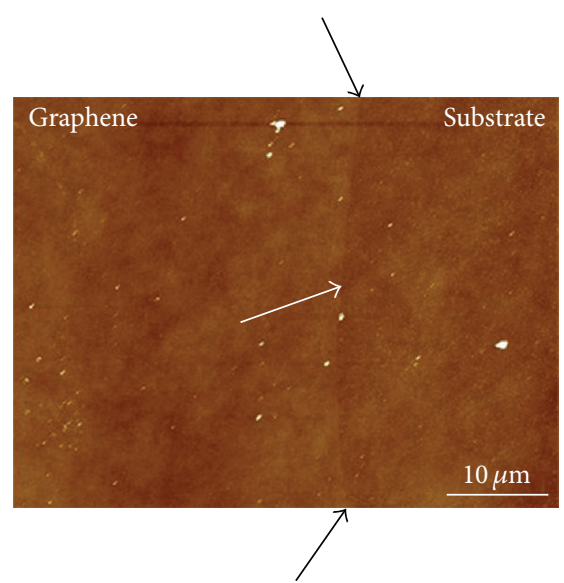

(a)

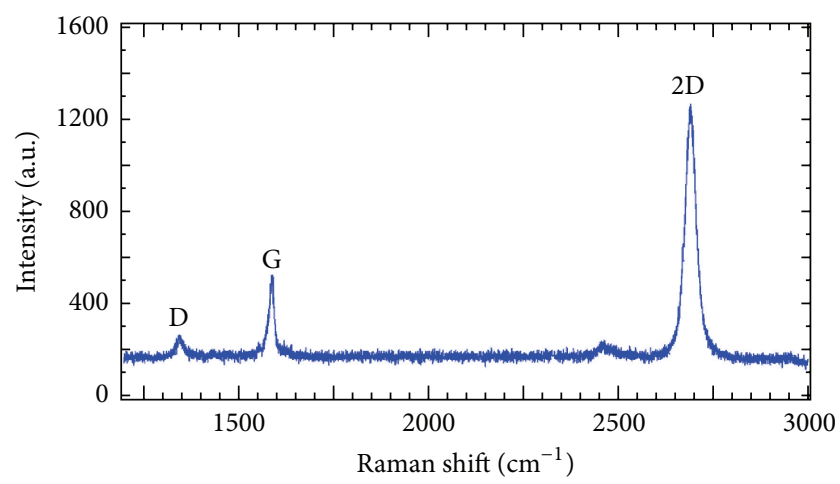

(b)

FIGURE 3: (a) Typical AFM image of the graphene transferred by the air-gap-assisted method. The edge of graphene is indicated by arrows. (b) Typical Raman spectrum (514 nm laser) of the graphene transferred by our method, pointing to a high lattice quality monolayer.

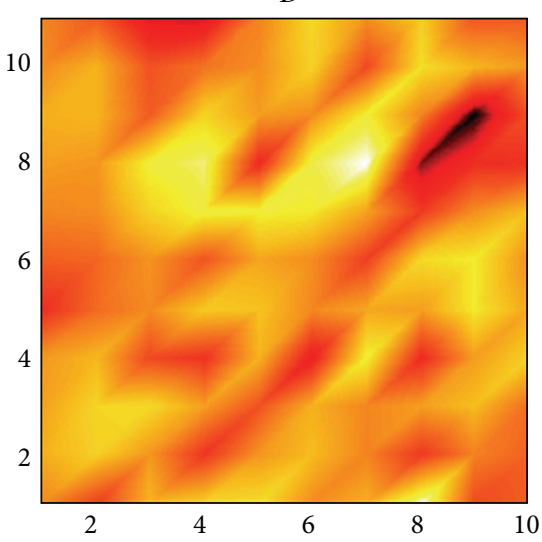

$2 \mathrm{D}$

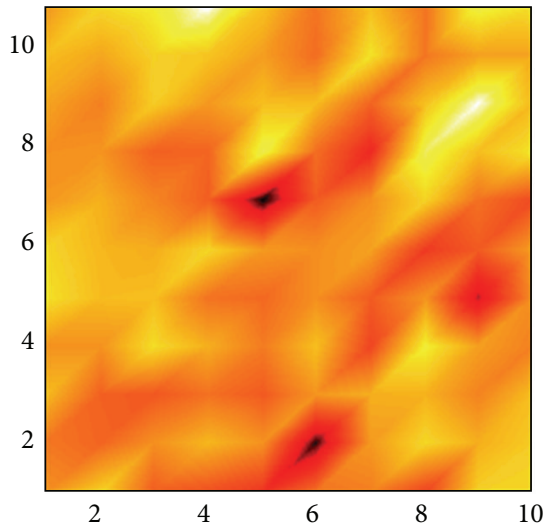

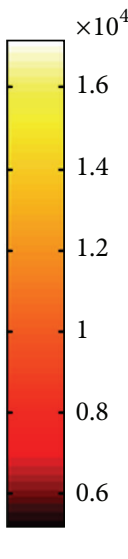

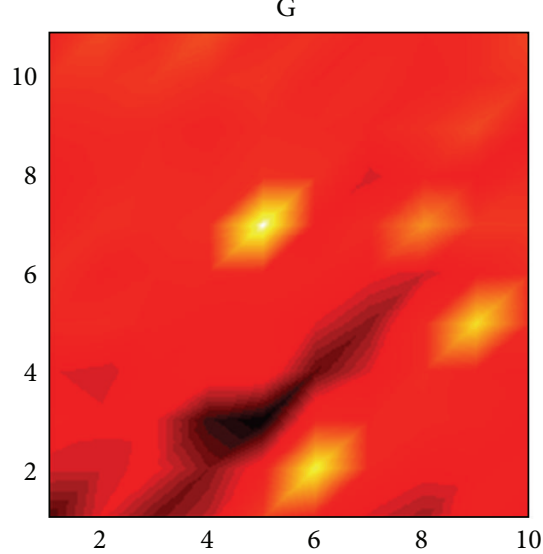

$\times 10^{4}$

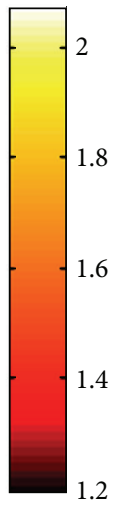

$2 \mathrm{D} / \mathrm{G}$

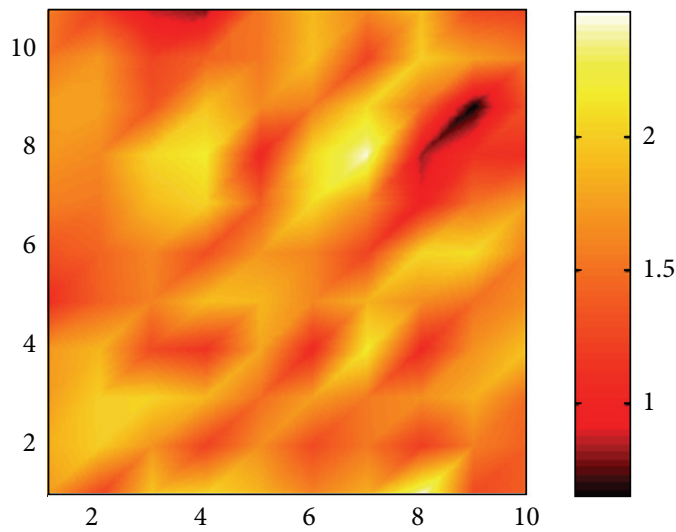

Figure 4: Raman mapping data of the transferred graphene on $\mathrm{SiO}_{2} / \mathrm{Si}$. In the four panels, $\mathrm{D}, \mathrm{G}, 2 \mathrm{D}$, and $2 \mathrm{D} / \mathrm{G}$ are plotted. The color bars for the intensities of the $\mathrm{D}, \mathrm{G}$, and $2 \mathrm{D}$ peaks use arbitrary units. The mapping area is a $10 \mu \mathrm{m} \times 11 \mu \mathrm{m}$ rectangle.

Figure 5(a) shows a photo of a graphene transistor fabricated by two-step photolithography. Again, the graphene is transferred by bubbling using a polymer support with encapsulated air gap to $\mathrm{SiO}_{2} / \mathrm{Si}$. In the first lithography, $15 \mathrm{~nm} \mathrm{Ti}$ and $50 \mathrm{~nm}$ Au electrodes are defined using lift-off technique. Before depositing the metals by sputtering, the corresponding $\mathrm{SiO}_{2}$ surface is already etched $65 \mathrm{~nm}$ deep to form a trench by dry etching. Hence, after metallization, the metal surface is almost of the same height as the surrounding unetched $\mathrm{SiO}_{2}$. We then transfer graphene to this substrate 


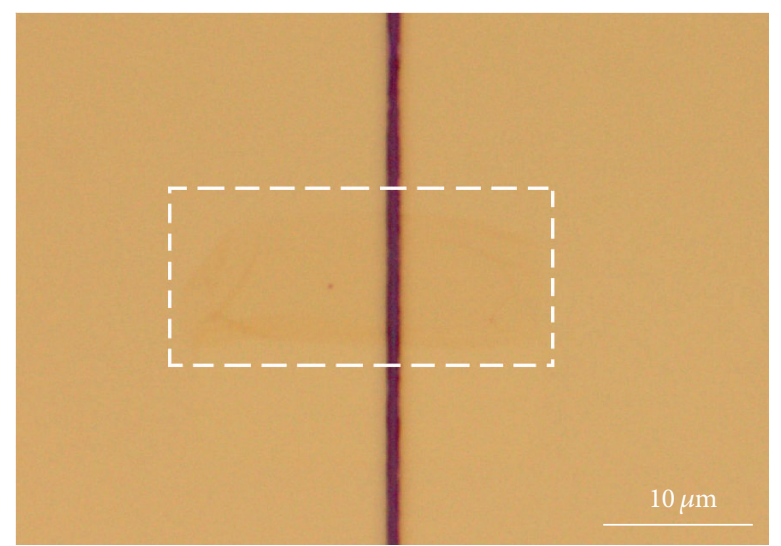

(a)

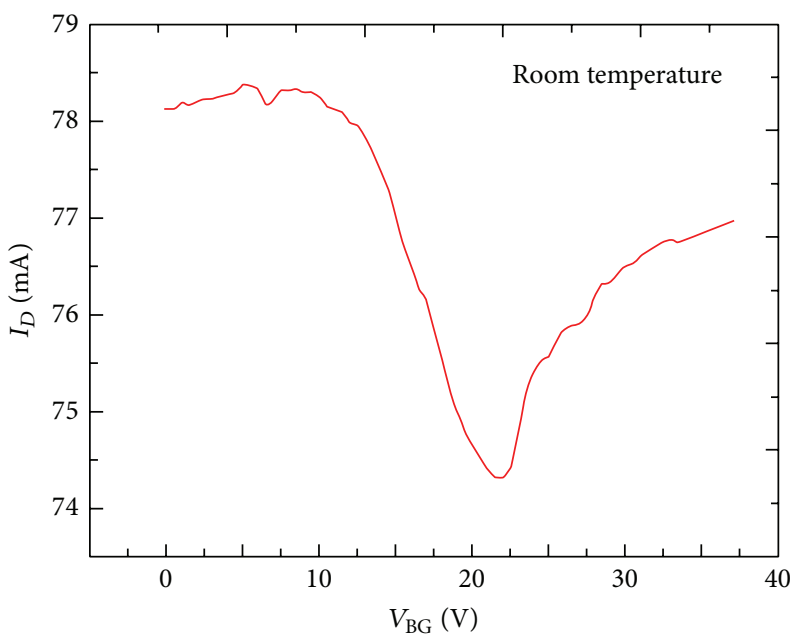

(b)

FIGURE 5: Room temperature electrical characterization of the graphene. (a) Optical micrograph of the transistor fabricated by photolithography. The yellow parts are the $\mathrm{Ti} / \mathrm{Au}$ source and drain. The graphene is attached on top of the $\mathrm{metals}(\mathrm{and}$ the $\mathrm{SiO} / \mathrm{Si})$, as indicated by the dashed line. The channel is $1 \mu \mathrm{m} \times 10 \mu \mathrm{m}$. (b) Channel current plotted against $V_{\mathrm{BG}}$ applied to the doped silicon. The graphene is slightly p-doped, as implied by the position of the Dirac point $\left(V_{\mathrm{BG}}=22 \mathrm{~V}\right)$.

with prepatterned electrodes. The graphene need not "climb up" the otherwise high metal electrodes, where the risk that the graphene breaks at the metal edges is reduced. In the second lithography, the graphene on top of metals is patterned to be a rectangle, as outlined in Figure 5(a). The transistor channel is $10 \mu \mathrm{m}$ wide and $1 \mu \mathrm{m}$ long. The doped silicon substrate is used as the back gate, which is capacitively coupled to the channel through $\sim 300 \mathrm{~nm} \mathrm{SiO}{ }_{2}$ dielectric. Figure 5(b) plots the channel current against gate voltage $V_{\mathrm{BG}}$ measured using a probe station at room temperature under ambient conditions, without any special treatment, for example, vacuum annealing. The graphene is somewhat $\mathrm{p}$ doped, as indicated by the position of Dirac point $\left(V_{\mathrm{BG}}=\right.$ $22 \mathrm{~V}$ ). Using a capacitor based model, the field effect mobility is estimated to be $\sim 3500 \mathrm{~cm}^{2} /(\mathrm{Vs})$. Although graphene processed with common photoresists can deteriorate [18], the value is still higher compared to our typical CVD graphene transferred by air-gap-free bubbling [12]. Therefore, we conclude that our transfer technique adds the least defects to the graphene and maximally preserves its intrinsic properties. Finally, we note that this is a scalable technology. The support layers are flexible, which could possibly be implemented into roll-to-roll electrochemical transfer line (the thickness for the PET foil and the plastic frame might need to be reduced). In that case where the sample is huge, to avoid the collapse of the PET foil, the plastic frame would have to be made into meshes, and the encapsulated air gaps were divided into many chambers. Also, the application can be readily extended to the transfer of 2D materials beyond graphene.

\section{Conclusions}

Summarily, we have developed a graphene mechanical supporting layer composed of PET foil/plastic frame/PMMA sandwich structure with an encapsulated air gap as the permeation stopping layer. Although the electrochemical transfer is of high industrial potential due to the excellent scalability, time- and cost-effectiveness, and ecofriendliness, the graphene is often damaged. This is because of the turbulence in the liquid but, more importantly, due to the mechanical distortion induced by the trapped bubbles in the sample center formed by the direct $\mathrm{H}_{2} \mathrm{O}$ and $\mathrm{H}^{+}$permeation through the polymer. Our supporting structure can hold the graphene firmly against the turbulence. Meanwhile, the PET foil and air gap stopping layer thoroughly prevent any particle permeation towards the graphene interfaces. As a result, the graphene damage is dramatically alleviated, as evidenced by the morphology and Raman and electrical characterization. Our facile method ultimately improves the controllability and reproducibility of the bubbling transfer, paving the way for its industrialization as the mainstream transfer technique for graphene and other two-dimensional materials.

\section{Competing Interests}

The authors declare that there are no competing of interests regarding the publication of this paper.

\section{Acknowledgments}

The authors acknowledge the support from Beijing Natural Science Foundation (4152003), National 863 Project (SS2015AA031004), Stiftelsen Olle Engkvist Byggmästare, Carl Tryggers Stiftelse, and STINT. They sincerely thank Professor Jianing Chen from the Institute of Physics, Chinese Academy of Sciences, and Mr. Songyu Li from Beijing University of Technology for their help in Raman measurement. 


\section{References}

[1] K. S. Novoselov, V. I. Fal'Ko, L. Colombo, P. R. Gellert, M. G. Schwab, and K. Kim, "A roadmap for graphene," Nature, vol. 490, no. 7419, pp. 192-200, 2012.

[2] S. Chandramohan, J. H. Kang, Y. S. Katharria et al., "Work-function-tuned multilayer graphene as current spreading electrode in blue light-emitting diodes," Applied Physics Letters, vol. 100, no. 2, Article ID 023502, 2012.

[3] E. Shi, H. Li, L. Yang et al., "Colloidal antireflection coating improves graphene-silicon solar cells," Nano Letters, vol. 13, no. 4, pp. 1776-1781, 2013.

[4] http://en.yibada.com/articles/17030/20150303/china-launches-world-s-first-graphene-phones-features-include-50.htm\# ixzz3ZX2H9tYi.

[5] T. Kobayashi, M. Bando, N. Kimura et al., "Production of a 100-m-long high-quality graphene transparent conductive film by roll-to-roll chemical vapor deposition and transfer process," Applied Physics Letters, vol. 102, no. 2, Article ID 023112, 2013.

[6] J. Sun, M. T. Cole, N. Lindvall, K. B. K. Teo, and A. Yurgens, "Noncatalytic chemical vapor deposition of graphene on hightemperature substrates for transparent electrodes," Applied Physics Letters, vol. 100, no. 2, Article ID 022102, 2012.

[7] X. Li, Y. Zhu, W. Cai et al., "Transfer of large-area graphene films for high-performance transparent conductive electrodes," Nano Letters, vol. 9, no. 12, pp. 4359-4363, 2009.

[8] Y. Wang, Y. Zheng, X. Xu et al., "Electrochemical delamination of CVD-grown graphene film: toward the recyclable use of copper catalyst," ACS Nano, vol. 5, no. 12, pp. 9927-9933, 2011.

[9] L. Gao, W. Ren, H. Xu et al., "Repeated growth and bubbling transfer of graphene with millimetre-size single-crystal grains using platinum," Nature Communications, vol. 3, article 699, 2012.

[10] C. J. Lockhart de la Rosa, J. Sun, N. Lindvall et al., "Frame assisted $\mathrm{H}_{2} \mathrm{O}$ electrolysis induced $\mathrm{H}_{2}$ bubbling transfer of large area graphene grown by chemical vapor deposition on $\mathrm{Cu}$," Applied Physics Letters, vol. 102, no. 2, Article ID 022101, 2013.

[11] Z. Zhan, J. Sun, L. Liu et al., "Pore-free bubbling delamination of chemical vapor deposited graphene from copper foils," The Journal of Materials Chemistry C, vol. 3, no. 33, pp. 8634-8641, 2015.

[12] J. Sun, Y. Nam, N. Lindvall et al., "Growth mechanism of graphene on platinum: surface catalysis and carbon segregation," Applied Physics Letters, vol. 104, no. 15, Article ID 152107, 2014.

[13] P. Sutandar, D. J. Ahn, and E. I. Franses, "FTIR ATR analysis for microstructure and water uptake in poly(methyl methacrylate) spin cast and Langmuir-Blodgett thin films," Macromolecules, vol. 27, no. 25, pp. 7316-7328, 1994.

[14] S. P. Koenig, L. Wang, J. Pellegrino, and J. S. Bunch, "Selective molecular sieving through porous graphene," Nature Nanotechnology, vol. 7, no. 11, pp. 728-732, 2012.

[15] X. Kun, X. Chen, D. Jun et al., "Graphene transparent electrodes grown by rapid chemical vapor deposition with ultrathin indium tin oxide contact layers for GaN light emitting diodes," Applied Physics Letters, vol. 102, no. 16, Article ID 162102, 2013.

[16] S. Hu, M. Lozada-Hidalgo, F. C. Wang et al., "Proton transport through one-atom-thick crystals," Nature, vol. 516, no. 7530, pp. 227-230, 2014.

[17] M. G. Walter, E. L. Warren, J. R. McKone et al., "Solar water splitting cells," Chemical Reviews, vol. 110, no. 11, pp. 6446-6473, 2010.
[18] R. Shi, H. Xu, B. Chen, Z. Zhang, and L.-M. Peng, "Scalable fabrication of graphene devices through photolithography," Applied Physics Letters, vol. 102, no. 11, Article ID 113102, 2013. 

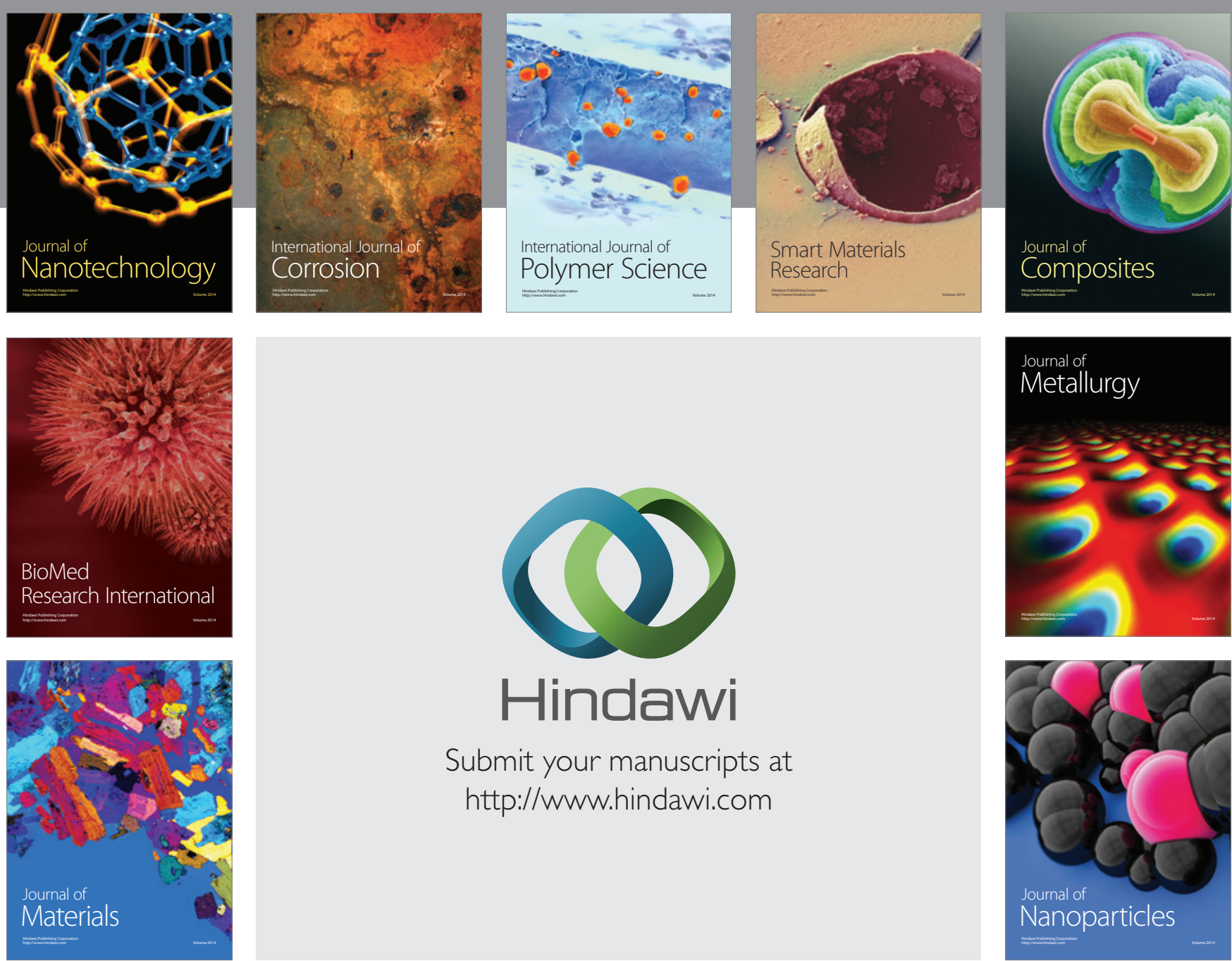

\section{Hindawi}

Submit your manuscripts at

http://www.hindawi.com

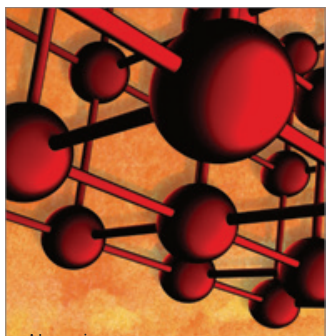

Materials Science and Engineering
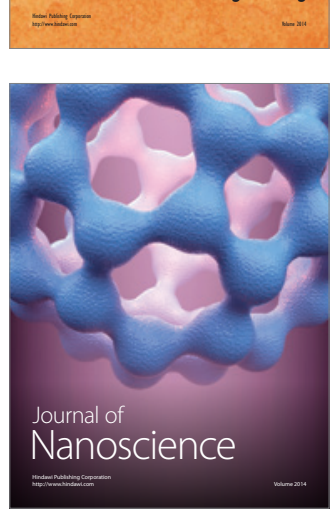
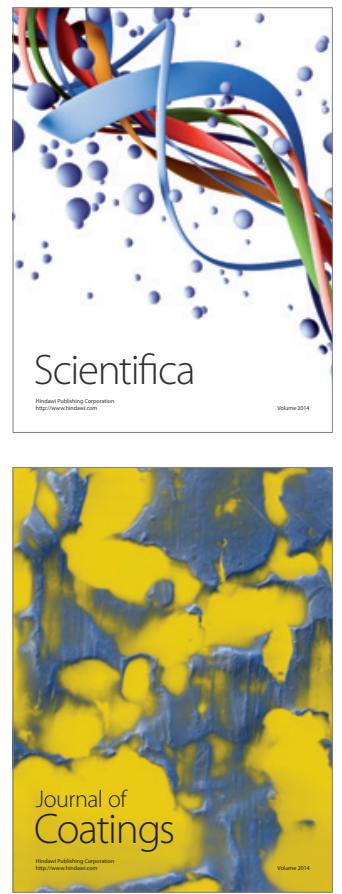
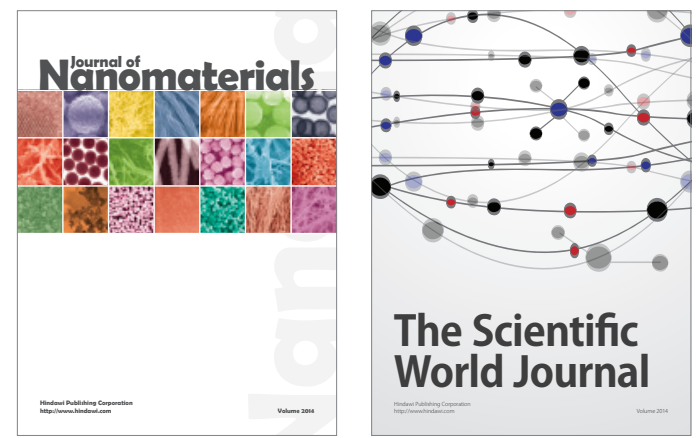

The Scientific World Journal
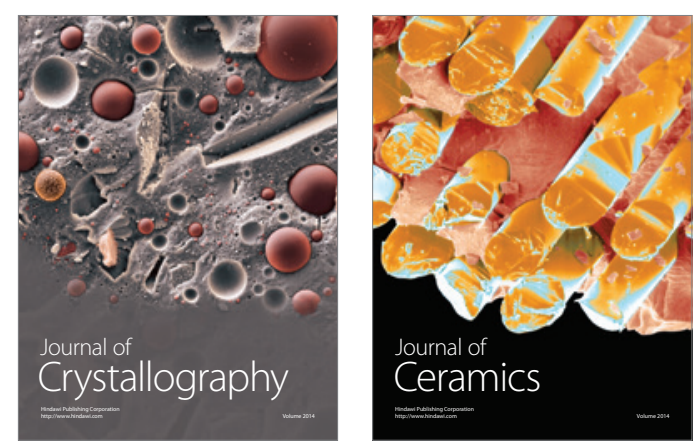
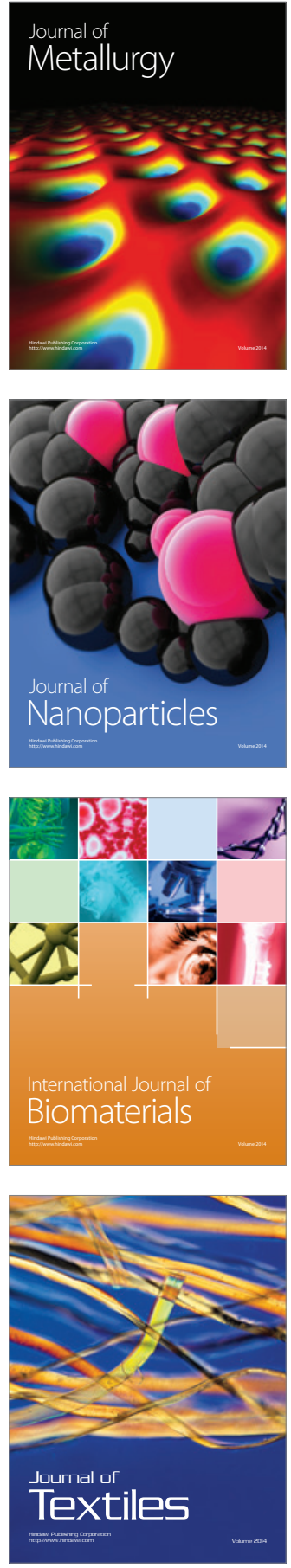